\title{
Synthesis and Electrical Characterization of Self-Supported Conducting Polypyrrole-Poly(vinylidene fluoride) Composite Films
}

\author{
Manish Taunk, Atul Kapil and Subhash Chand*
}

Department of Applied Sciences, National Institute of Technology, Hamirpur, Himachal Pradesh, PIN: 177 005, India

\begin{abstract}
We report the synthesis of polypyrrole composite films by chemical oxidation polymerization of pyrrole diffused inside poly(vinylidene fluoride) matrix and temperature dependant dc conductivity study of these composite films. Ammonium persulfate is used as an oxidizing agent and $\mathrm{HCl}$ as the dopant in aqueous medium. This technique produces highly conductive, flexible and free standing polymer composite films in semi-interpenetrating architecture. It is observed that conductivity of the composite films can be controlled by varying the dopant concentration. The variation of conductivity with temperature indicates the semiconducting nature of these composite films. The temperature dependence of conductivity is explained in the light of two different models from which activation energy $\left(E_{a}\right)$ and other hopping parameters are determined. Experimental data fits well with variable range hopping model and three dimensional charge transport mechanism is shown to occur in these films.
\end{abstract}

Keywords: Polypyrrole, conducting polymer thin films, electrical characterization, conductivity, activation energy.

\section{INTRODUCTION}

Electronically conducting polymers have been the subject of great interest to chemists and physicists in recent years. Because of the large number of possible applications of these materials in various electronic devices such as electrochromic displays (ECD), light emitting diodes (LEDs), field effect transistors (FETs), chemical sensors, etc. there has been considerable interest to replace conventional inorganic semiconductors by their organic counterparts. Organic semiconductors have some unique properties and versatility like light weight, flexibility, corrosion resistivity and ease of processing. After the discovery of highly conductive $\left(10^{3} \mathrm{Scm}^{-1}\right)$ iodine doped polyacetylene $(P A)$ in 1977 [1], variety of new conducting polymers such as polypyrrole $(P P y)$, polythiophene $(P T h)$, polyaniline $(P A n i)$, polyfuran $(P F u)$ etc. have been prepared and extensively studied for their chemical, morphological, optical and electronic properties [2-5].

The common feature in the structure of conducting polymers is poly-conjugation of the $\pi$-system of their backbone [4]. Among these conducting polymers, PPy and its derivatives are of particular interest because of their high electrical conductivity and stability in the doped state. It is well known that PPy can be prepared by electrochemical and chemical oxidation methods $[4,6]$. Both the polymerization methods yield conducting $P P y$ which exhibits good stability under ambient conditions. The chemical oxidative polymerization of pyrrole results in black powder and reaction mechanism has been known for many years [7-10]. During oxidative polymerization, pyrrole $(P y)$ monomer looses a proton at $\alpha$ position and get linked with other monomer of

*Address correspondence to this author at the Department of Applied Sciences, National Institute of Technology, Hamirpur, Himachal Pradesh, PIN: 177 005, India; Tel: +91-1972-254136, +91-1972-254137, +919418353397; E-mail: schand@nitham.ac.in,mtaunkz@gmail.com pyrrole to form the long chain of polymer PPy as shown in Scheme 1.

Several researchers have reported the electrical conductivity of chemically synthesized $P P y$ with maximum conductivity upto $500 \mathrm{Scm}^{-1}$, which is an appreciable value for most of the applications [11-17]. The PPy prepared in these reports is in bulk pellet form which exhibits poor mechanical properties, e.g., brittle in nature and low level of processability which hinders its preparation in thin film form for its applications in solid state devices and electronics [18]. Studies have been done to increase the processability by chemically altering the monomer or dopants used $[19,20]$. In addition to chemical modification, various attempts have been made in the past to improve the processability and hence application part by preparing blends and composites containing PPy [2123]. Improvements are expected from composites in which conducting polymers can be dispersed in the insulating polymer matrix which provides a flexible, large area substrate for electronic properties. Composites of conducting $P P y$ prepared by electrochemical and chemical methods with other insulating polymers like polydimethyl siloxane [24], polyindene [25], polyimide [26], polypropylene [27], polyvinylchloride [28], polyoxyphenylene [29], polymethylmethacrylate [30], polyvinylidene fluoride $(P V D F)[31,32]$ are reported in the literature. These composite materials are characterized for their electrical, thermal, chemical and mechanical properties. $P P y$ and $P V D F$ composite are also reported in various configurations for their applications as actuators [33], metal ion transport across composite membranes [34] and hydrogen generating catalysts [35]. These composite polymer films are prepared by electrochemical method.

The goal of present work is to develop self supported flexible conducting polymer films with good electrical conductivity. In this study we have prepared free standing conducting composite films by chemical oxidative polymeriza- 


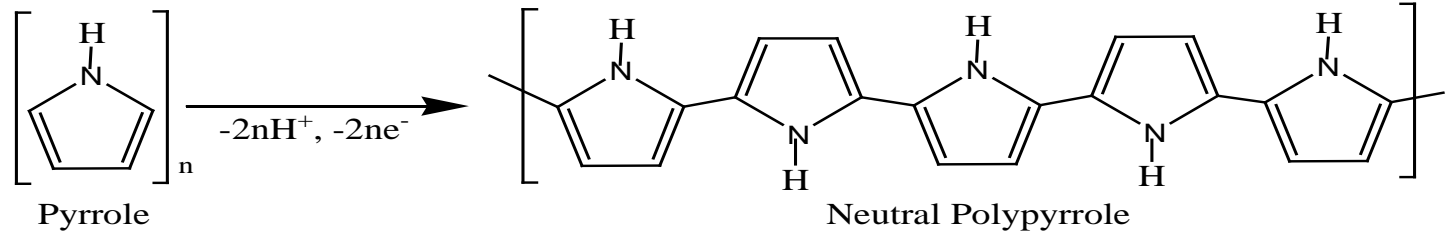

Scheme 1. Chemical Oxidative polymerization of Pyrrole.

tion of pyrrole soaked in $P V D F$ matrix which leads to a interpenetrating network of conducting $P P y$ and insulating $P V D F$ polymer chains. In this way continuous channels of conducting polymer $P P y$ were formed within $P V D F$ matrix. The main advantage of this method is that it does not require additional conducting substrates as a support to hold $P P y$ thin films. Needless, to say that the $\mathrm{HCl}$ doped PPy can't be prepared in film form because of being insoluble in organic solvents. $P V D F$ is used as an insulating polymer because of its good mechanical flexibility, high thermal and chemical stability.

\section{EXPERIMENTAL}

\section{Materials}

Pyrrole (Py) monomer (Spectrochem India), polyvinylidene fluoride $(P V D F)$ and ammonium persulfate, $\left(\mathrm{NH}_{4}\right)_{2} \mathrm{~S}_{2} \mathrm{O}_{8}$ (APS) provided by Alfa aesar were used as the starting material for the preparation of composites in this study. Methanol, $\mathrm{HCl}$ and dimethyl formamide (DMF) of reagent grade were purchased from Merck.

\section{Preparation of Films}

In this study oxidative polymerization of monomer pyrrole is allowed inside the $P V D F$ matrix. $P V D F$ is mixed with dimethyl formamide $(D M F)$ and kept under continuous stirring until a homogeneous solution is formed. The solution was then cast in covered flat glass petri dish and dried in vacuum oven at $50{ }^{\circ} \mathrm{C}$. After evaporation of solvent, free standing insulating films of $P V D F$ were obtained. These films were cut into strips and immersed in pyrrole monomer to allow the monomer diffusion inside the $P V D F$ matrix. The excessive monomer on the $P V D F$ film surface was removed by blotting with filter paper to occur polymerization only inside the $P V D F$ matrix. The pyrrole absorbed $P V D F$ films were then immersed in aqueous solution of $\mathrm{HCl}$ (as dopant) and $A P S(0.5 \mathrm{M})$ as an oxidizing agent at room temperature. Color of composite films found to change from light green to dark blue and finally black which shows progressive oxidative polymerization of pyrrole monomers. Different molar concentrations of $\mathrm{HCl}$ were used to make polymer composite films of varying conductivity. After polymerization for about four hours films were taken out of aqueous solution and washed several times with de-ionized water $(18.2 \mathrm{M} \Omega$ ) and methanol to remove un-reacted traces. Thereafter these films were dried under vacuum and then stored in vacuum desiccators for later characterization. By this method $P P y-P V D F$ composite films with different dopant concentration were obtained. The resulting polymer composite films are smooth, flexible and can be rolled like paper.

\section{Electrical Characterization}

The electrical resistivity measurements of the polymer composite films were made using the standard four probe technique, where electrical contacts were established through four collinear and equally spaced pointed probes. The electrical resistivity $(\rho)$ can be defined as

$\rho=\frac{2 \pi s}{C . F .}\left[\frac{V}{I}\right]$

where $I, V$ and $s$ represents applied current through outer probes, voltage produced between inner probes and probe spacing, respectively. C.F. $=2 \log 2(\mathrm{~s} / \mathrm{d})$ is correction factor which depends upon film thickness $(d)$ and the probe spacing $(s)$.

Film thickness was measured using digital micrometer and found to be $\sim 40 \mu \mathrm{m}$ for all samples. The conductivity measurement of these films is also done with Van-Der Pauw method in square geometry in which film contacts were made at the corners of sample using conducting silver paste. The conductivity data measured using two methods were found to be in good agreement. The electrical measurements were performed using programmable current source (Keithley source meter 2400) and an electrometer (Keithley 6514) for voltage measurement using a computer controlled GPIB data acquisition system. A computer programme in visual basic was developed to record automated $I-V$ data on computer. The measurement were made in the temperature range of $298 K-373 K$.

\section{RESULTS AND DISCUSSION}

Doped conjugated polymers are also termed as synthetic metals because of their high conductivity comparable to that of the metals. However, for most of the conducting polymers temperature coefficient of conductivity is opposite to that of metals i.e. conductivity of these polymers increases with increase in temperature, which is a characteristic of semiconductors. During the polymerization process dopant $\mathrm{HCl}$ breaks up into parts $\left(\mathrm{H}^{+}\right)$and counter ions $\left(\mathrm{Cl}^{-}\right)$which diffuses in to the polymeric chain. Dopants in case of conducting polymers do not replace the monomer position but they are trapped in interstitial sites and stabilise charges along the backbone permitting higher conductivity.

When electric field is applied across the sample, localized motion of the trapped charges acts as effective electric dipoles. Short range distortions due to these dipoles leads to the formation of polarons and bipolarons. When an electron is removed from a pyrrole ring in polymer chain, a free radical and positively charged ion is produced. The radical ion and cation are coupled to each other and complex is known as polaron. In order to maintain the charge neutrality of the polymer, anion must be present there to compensate the cationic polaron. Upon further oxidation of polaron the free radical is removed and a species containing positive charge at both ends is formed which is known as bipolaron. Polarons and bipolarons in PPy when doped with chloride ions are shown in Scheme 2. These polarons and bipolarons are the 
<smiles></smiles><smiles>Cc1ccc([C-]2C=C/C(=C3/C=CNN3)N/C2=C2\C=CNN2)[nH]1</smiles>

Scheme 2. Polaron and Bipolaron formation in Polypyrrole upon chloride doping.

main charge carriers in organic materials and are responsible for their electronic properties.

\section{Current-Voltage Characteristics}

The $I-V$ characteristics of $P P y-P V D F$ composite films for all samples were measured at different temperature and found to be linear in all the cases as shown in Figs. (1 \& 2). This linearity of I-V characteristics is an indication of Ohmic nature of composite films. In this study $P P y-P V D F$ composite films were obtained with four different concentrations of $\mathrm{HCl}$.

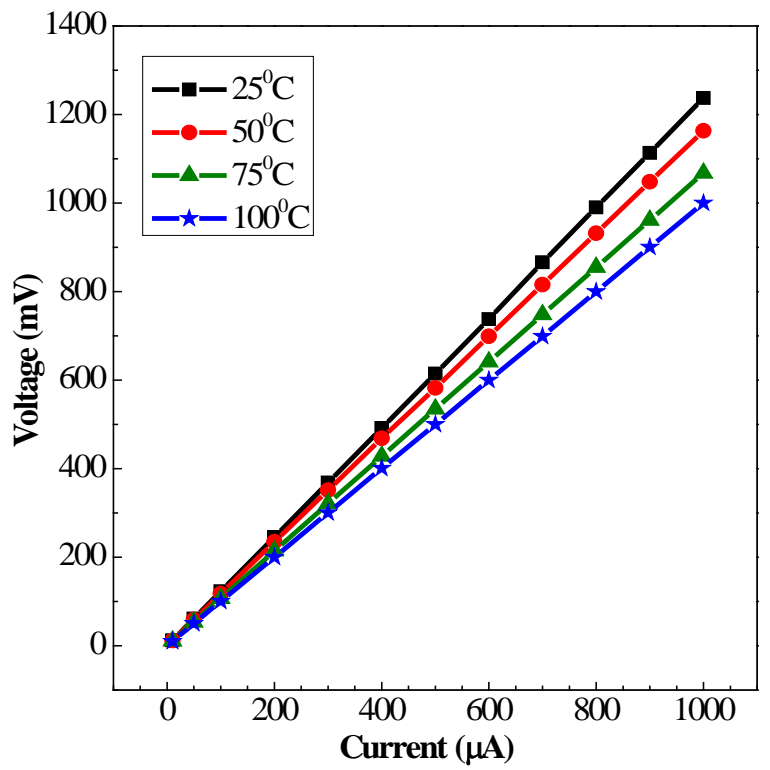

Fig. (1). I-V Characteristics of PPy-PVDF composite film at different temperatures for $0.1 \mathrm{M} \mathrm{HCl}$ doped.
The conductivity (reciprocal of resistivity) of these composite polymer films was estimated by four probe method using equation (1) and the values thus obtained are shown in Table 1 at various temperatures and doping concentrations. It is obvious from Table $\mathbf{1}$ that the conductivity increases with rise in temperature for all samples. The increase in conductivity is more for highly doped samples. This increase in conductivity with rising temperature rules out the possibility of considering these films to be synthetic metal and indicates their semiconducting nature.

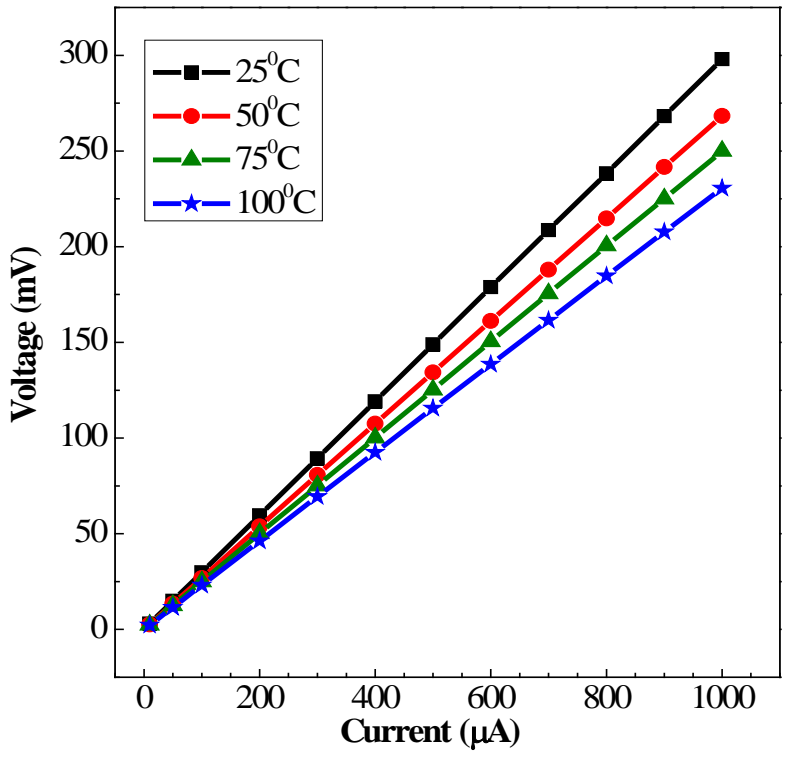

Fig. (2). I-V Characteristics of PPy-PVDF composite film at different temperatures for $0.5 \mathrm{M} \mathrm{HCl}$ doped.

Table 1. Electrical Conductivity of PPy Composite Films at Different Temperatures

\begin{tabular}{|c|c|c|c|c|}
\hline Temperature & \multicolumn{4}{|c|}{ Electrical conductivity of $P P y$ composite films $\left(\mathrm{Scm}^{-1}\right)$} \\
\hline $25^{\circ} \mathrm{C}$ & $1.96 \times 10^{-2}$ & $2.4 \times 10^{-2}$ & $8.0 \times 10^{-2}$ & $1.33 \times 10^{-1}$ \\
\hline $50^{\circ} \mathrm{C}$ & $2.03 \times 10^{-2}$ & $2.55 \times 10^{-2}$ & $9.23 \times 10^{-2}$ & $1.41 \times 10^{-1}$ \\
\hline $75^{\circ} \mathrm{C}$ & $2.22 \times 10^{-2}$ & $2.79 \times 10^{-2}$ & $1.0 \times 10^{-1}$ & $1.5 \times 10^{-1}$ \\
\hline
\end{tabular}


This variation in conductivity with doping concentration is shown in Fig. (3). It is evident from Fig. (3) that with the increase in concentration of $\mathrm{HCl}$, the conductivity of these composite films increases. This is due to the increase in the number of polarons created. Electrical conductivity values calculated for these four samples at four different temperatures are given in Table $\mathbf{1 .}$

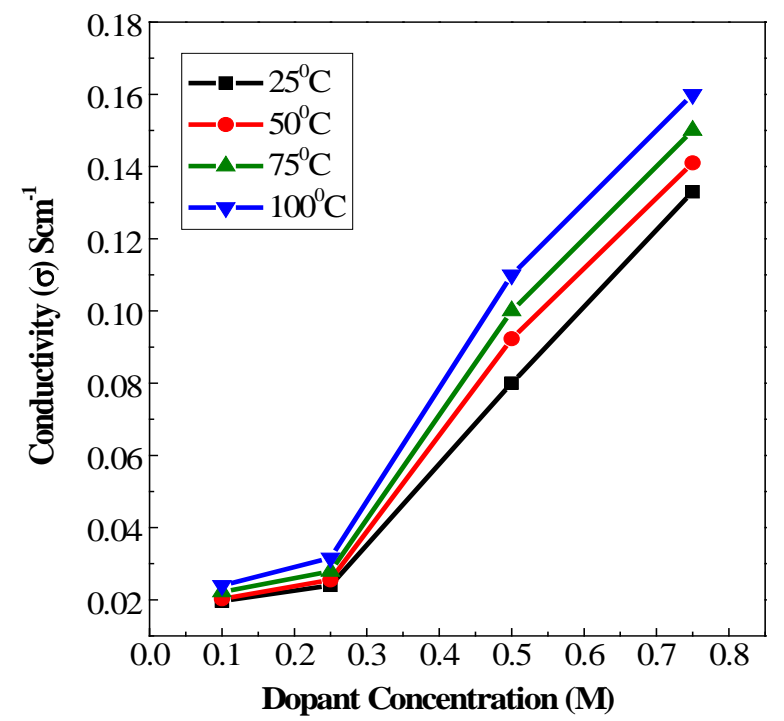

Fig. (3). Variation of electrical conductivity of composite films with dopant concentration.

\section{Temperature Dependant Conductivity}

In order to determine the current conduction mechanism in $P P y-P V D F$ composite films, the temperature dependence of electrical conductivity was studied in the temperature range from $298 K$ to $373 K$. The electrical conductivity behavior can be explained using two different models.

\section{Arrhenius Model}

Logarithmic plot of $d c$ conductivity as a function of reciprocal temperature is shown in Fig. (4). This type of electrical conductivity behavior follows Arrhenius model [36] and according to this model conductivity temperature relation is

$\sigma=\sigma_{0} \exp \left[\frac{-E_{a}}{k T}\right]$

Where $\sigma_{0}$ is constant pre-exponential factor, $k$ is the Boltzmann constant and $E_{a}$ is the carrier activation energy. Solid black line represents the best linear fit of experimental data with equation (2), from this straight line fit of experimental data we can infer that the conductivity exhibits a thermally activated process in the measured temperature range.

Carrier activation energy can be calculated from the slopes (equal to $E_{a} / 1000 k$ ) of these plots. The activation energies obtained for these films are $35.5 \mathrm{meV}$ for $0.1 \mathrm{M}$ and $31.2 \mathrm{meV}$ for $0.5 \mathrm{M}$. The activation energies of our samples differs slightly from the activation energies reported in case of pure $P P y$ [37] and electrochemically synthesized $P P y$
[38]. The difference in activation energies can be attributed to the different dopants used. However, in our studies the lower activation energy for higher dopant concentration sample may be due to creation of bipolaron bands within the HOMO (highest occupied molecular orbital)- LUMO (lowest unoccupied molecular orbital) bandgap [10].

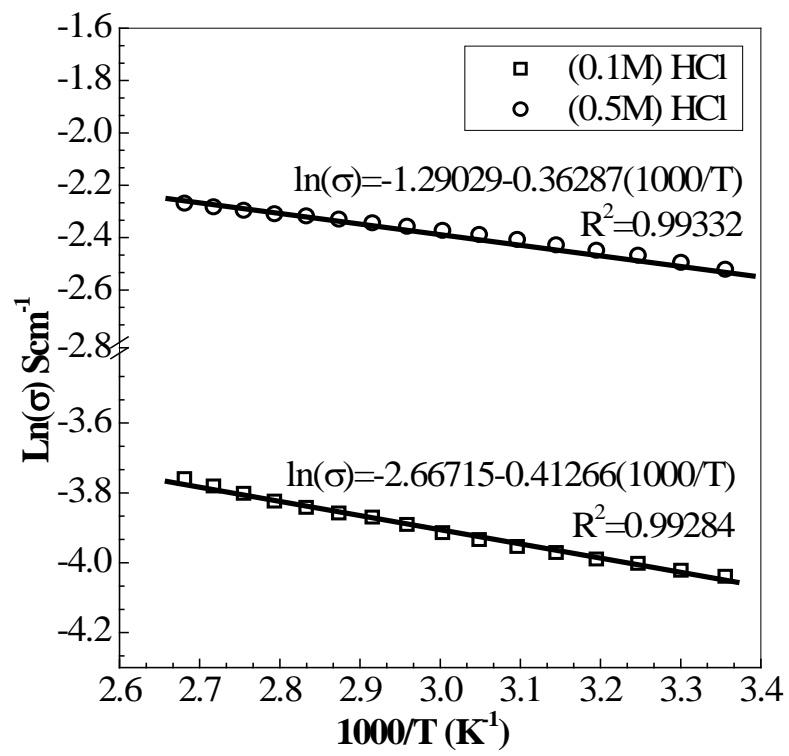

Fig. (4). Plot of $\operatorname{Ln}(\sigma)$ Vs 1000/T for PPy composite films doped with $\mathrm{HCl}$. Solid line represent linear fit to experimental data.

\section{Variable Range Hopping Model}

Since disorder plays an important role in conducting polymers, Variable Range Hopping ( $V R H)$ is also considered as one of the major carrier transport mechanism, which is originally proposed for inorganic amorphous semiconductors. Hopping refers to tunneling transitions from occupied to unoccupied localized states and is based upon assumption that energy difference is maintained by emission or absorption of one or more phonons. The temperature dependence of conductivity in organic semiconductors is generally described by Mott's VRH mechanism which includes the phonon assisted quantum mechanical transport phenomenon for the movement of charge carriers. In this type of conduction hopping distance is not constant. According to this model the characteristic temperature dependence of conductivity is given by [39]

$\sigma=\sigma_{0} \exp \left[\frac{-T_{0}}{T}\right]^{\gamma}$

where $\sigma_{0}$ is high temperature limit of conductivity, $T_{0}$ is Mott's characteristic temperature associated with degree of localization of the electronic wave function and the exponent $\gamma=1 /(1+d)$ determines the hopping space dimensionality of the charge transport in conducting medium. Value of $(d=3$, $2,1)$ for three, two and one dimensional hopping transport respectively.

In order to evaluate the possibility of $V R H$ and dimensionality of conduction process in polymeric films semi logarithmic plot of conductivity as a function of $(1 / T)^{1 / 4}$ should follow a straight line according to equation (3). The experimental values of $P P y$ film conductivity have been plotted 


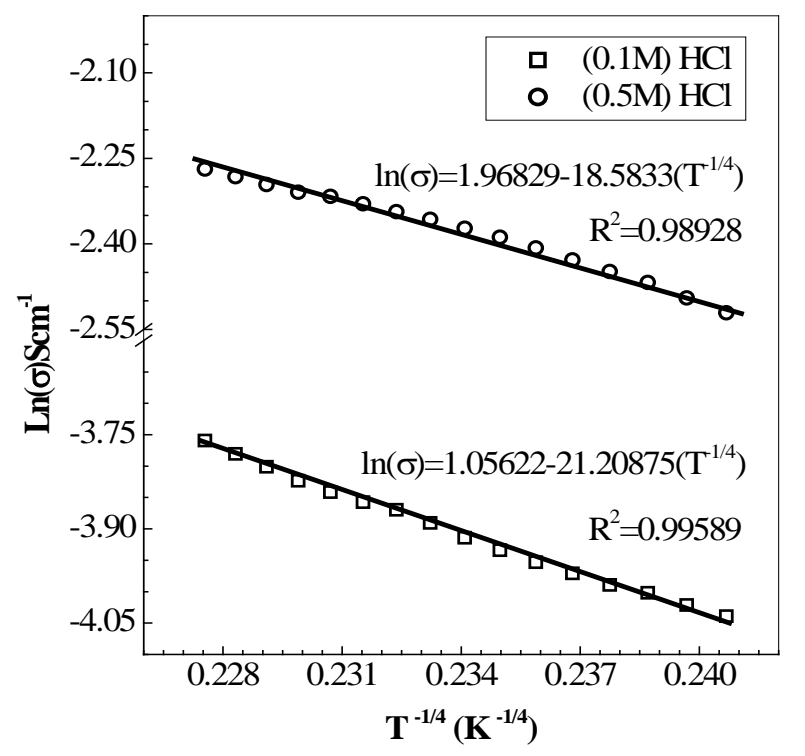

Fig. (5). Plot of $\operatorname{Ln}(\sigma)$ Vs $T^{(-1 / 4)}$ for PPy composite films doped with $\mathrm{HCl}$. Solid line represent linear fit to experimental data.

against $(1 / T)^{1 / 4}$ in Fig. (5) which is found to fit in a straight line. It is evident from the Fig. (5) that Mott's law is obeyed over entire temperature range and indicates $3 d$ charge transport in these samples.

For three dimensional transport hopping parameters to be given by[39] are

$T_{0}=\left[\frac{18}{r_{0}^{3} k N\left(E_{F}\right)}\right]$

where $r_{0}$ is localization length, $k$ is Boltzmann constant, $N\left(E_{F}\right)$ is density of states at Fermi level.

Hopping distance $(R)$ and average hopping energy $(W)$ were defined by Mott and Davis as

$R=\left[\frac{9 r_{0}}{8 \pi N\left(E_{F}\right) k T}\right]^{1 / 4}$

and

$W=\left[\frac{3}{4 \pi R^{3} N\left(E_{F}\right)}\right]$

Bredas et al. [40], have reported that for $P P y$ hopping state is delocalized over four pyrrole rings, so it is assumed

Table 2. The Values of Variable Range Hopping Parameters

\begin{tabular}{|c|c|c|}
\hline VRH Parameters & $(\mathbf{0 . 1 M})$ Sample & (0.5M) Sample \\
\hline \hline $\mathbf{T}_{\mathbf{0}}{ }^{\mathbf{1 / 4}}\left(\mathbf{K}^{\mathbf{1 / 4}}\right)$ & 21.2 & 18.58 \\
\hline $\mathbf{T}_{\mathbf{0}}(\mathbf{K})$ & 202301.4 & 119251.4 \\
\hline $\mathbf{N}\left(\mathbf{E}_{\mathbf{F}}\right)\left(\mathbf{e V}^{-1} \mathbf{c m}^{-\mathbf{1}}\right)$ & $1.032 \times 10^{21}$ & $1.751 \times 10^{21}$ \\
\hline $\mathbf{R}(\stackrel{\AA}{)})$ & 19.43 & 17.02 \\
\hline $\mathbf{R} / \mathbf{r}_{\mathbf{0}}$ & 1.94 & 1.7 \\
\hline $\mathbf{W}(\mathbf{m e V})$ & 31.5 & 27.6 \\
\hline
\end{tabular}

that localization length $\left(r_{0}\right)$ will be of the order of $10 \AA$ for the monomer dimension of $\sim 3 \AA$. The hopping parameters were determined from the experimental data plotted in Fig. (5) using equations (4-6) and are given in Table $\mathbf{2}$. Here average hopping distance $(R)$ and average hopping energy $(W)$ are calculated at room temperature.

The parameter $T_{0}$, has larger value for lower doped sample and also its $N\left(E_{F}\right)$ value is less as compared to the highly doped sample. Also in the $0.5 \mathrm{M}$ doped sample $R$ is decreased by nearly one monomer unit. It is required for $V R H$ conduction process that $R / r_{0} \gg 1$ and $W \gg k T$. In these samples it is observed that both the conditions are satisfied in the studied temperature range. The values of $R$ varies from $19.4 \AA$ to $18.3 \AA$ for $0.1 \mathrm{M}$ doped sample and from $17.02 \AA$ to $16.09 \AA$ for $0.5 \mathrm{M}$ doped sample in the measured temperature range, similarly $W$ varies from $31.5 \mathrm{meV}$ to $37.3 \mathrm{meV}$ for $0.1 \mathrm{M}$ doped sample and from $27.6 \mathrm{meV}$ to $32.7 \mathrm{meV}$ for $0.5 \mathrm{M}$ doped sample. This type of behavior may be due to increase in the disorder of system with increase in temperature, so that conduction takes place by hopping of carriers to states located close to initial state and results in decreased $R$ value. Similarly $W$ increases because with the increase in disorder more energy is required by carrier to make a transition between two states in the hopping process.

\section{CONCLUSION}

In this work we have reported the synthesis and electrical properties of flexible and free standing $P P y-P V D F$ composite films with room temperature conductivity ranging from $1.96 \times 10^{-2}$ to $1.33 \times 10^{-1} \mathrm{Scm}^{-1}$ for different doping concentrations of $\mathrm{HCl}$. Increasing the dopant concentration give rise to increase in conductivity of these films. The $d c$ electrical conductivity of the composite polymer films has been investigated in the temperature range from $298 K$ to $373 K$ and it was observed that films are semiconducting in nature. The conduction mechanism follows the Arrhenius model as well as Mott's variable range hopping model in the studied temperature range. Activation energy of the carriers obtained is found to be of the order of few milli electron volts. The density of states at Fermi level, average hopping distance and hopping energy have been calculated and various aspects related to these are discussed. Three dimensional charge transport mechanism as suggested by Mott's VRH model is shown to be present in these polymer composite thin films. These flexible and free standing polymer films with semiconducting nature will be promising material for development of foldable and large area displays and devices.

\section{REFERENCES}

[1] Shirakawa, H.; Louis, E.J.; MacDiarmid, A.G.; Chiang, C.K.; Heeger, A.J. Synthesis of electrically conducting organic polymers: halogen derivatives of polyacetylene, $(\mathrm{CH}) x$. J. Chem. Soc. Chem. Commun., 1977, 578.

[2] Greene, R.L.; Street, G.B.; Sutude, L.J. Superconductivity in Polysulfur Nitride (SN)x. Phys. Rev. Lett., 1975, 34, 577-579.

[3] Bredas, J.L.; Themans, B.; Fripiat, J.G.; Andre, J.M.; Chance, R.R. Highly conducting polyparaphenylene, polypyrrole, and polythiophene chains: An ab initio study of the geometry and electronic-structure modifications upon doping. Phys. Rev. B, 1984, 29, 6761-6773.

[4] Skotheim, T.A. Handbook of Conducting Polymers, Marcel Dekker, New York, 1986; vol. 1\&2.

[5] Skorobogatov, V.M.; Krivoshei, J.V. The structure and properties of highly conducting polyacetylene complexes. Russ. Chem. Rev., 1988, 57, 461-474. 
[6] Yamaura, M.; Hagiwara, T.; Iwata, K. Enhancement of electrical conductivity of polypyrrole film by stretching: Counter ion effect. Synth. Met., 1988, 26, 209-224.

[7] Armes, S.P. Optimum reaction conditions for the polymerization of pyrrole by iron(III) chloride in aqueous solution. Synth. Met., 1987, 20, 365-371.

[8] Bunting, R.K.; Swarat, K.; Yan, D. Synthesis and characterization of a conduction polymer: An Electrochemical experiment for general chemistry. J. Chem. Educ., 1997, 74, 421-423.

[9] Henry, M.C.; Hsueh, C.C.; Timko, B.P.; Freund, M.S. Reaction of pyrrole and chlorauric acid. J. Electrochem. Soc., 2001, 148, D155D162.

[10] Shaktawat, V.; Jain, N.; Saxena, R.; Saxena, N.S; Sharma, K.; Sharma, T.P. Temperature dependance of electrical conduction in pure and doped polypyrrole. Polym. Bull., 2006, 57, 535-543.

[11] Pron, A.; Kucharski. Z.; Dehe, G.; Lefrant, S.; Suwalski, J. Mössbauer spectroscopy studies of selected conducting polypyrroles. J. Chem. Phys., 1985, 83, 5923.

[12] Kanazawa, K.K.; Geiis, R.H.; Gill, W.D.; Street, G.B.; Rabolt, J.F. 'Organic metals': polypyrrole, a stable synthetic 'metallic' polymer. J. Chem. Soc. Chem. Commun., 1979, 854.

[13] Diaz, A.; Castillo, J.I.; Logan, J.A.; Lee, W.Y. Electrochemistry of conducting polypyrrole films. J. Electroanal. Chem., 1981, 129, 115-132.

[14] Machida, S.; Miyata, S.; Techagumpuch, A. Chemical synthesis of highly electrically conductive polypyrrole. Synth. Met., 1989, 31, 311-318.

[15] Dubitsky, Y.A.; Zhubanov, B.A.; Maresch, G.G. Synthesis of polypyrroles in the presence of ferric tetrafluoroborate. Synth. Met., 1991, 41, 373-376.

[16] Singh, R.; Tandon, R.P.; Panwar, V.S.; Chandra, S. Origin of d.c. conduction and dielectric relaxation in lightly doped polypyrrole films. Thin Solid Films, 1991, 196, L15-L20.

[17] Vernitskaya, T.V.; Efimov, O.N. Polypyrrole: a conducting polymer; its synthesis, properties and applications. Russ. Chem. Rev., 1997, 66, 443-457.

[18] Diaz, A.F.; Lacroix, C. Synthesis of electroactive/conductive polymer films: electrooxidation of heteroaromatic compounds. New J. Chem., 1988, 12, 171-180.

[19] Lee, J.Y.; Kim, D.Y.; Kim, C.Y. Synthesis of soluble polypyrrole of the doped state in organic solvents. Synth. Met., 1995, 74, 103106.

[20] Shen, Y.; Wan, M. In situ doping polymerization of pyrrole with sulfonic acid as a dopant. Synth. Met., 1998, 96, 127-132.

[21] Bhattacharya, S.; Saha, S.K.; Chakrovorty, D. Conductivity relaxation behavior of interpenetrating polymer network composites of polypyrrole and poly(styrene-co-butyl acrylate). J. Polym. Sci. B: Polym. Phys., 2000, 38, 1193-1200.

[22] Bhat, N.V.; Gadre, A.P.; Bambole, V.A. Structural, mechanical, and electrical properties of electropolymerized polypyrrole composite films. J. Appl. Polym. Sci., 2001, 80, 2511-2517.

[23] Tang, M.; Wen, T.Y.; Du, T.B.; Chen, Y.P. Synthesis of electrically conductive polypyrrole-polystyrene composites using super- critical carbon dioxide: II. Effects of the doping conditions. Eur. Polym. J., 2003, 39, 151-156.

[24] Cakmak, G.; Kucukyavuz, Z.; Kucukyavuz, S.; Cakmak, H. Mechanical, electrical and thermal properties of carbon fiber reinforced poly(dimethylsiloxane)/polypyrrole composites. Composites Part A, 2004, 35, 417-421.

[25] Bozkurt, A.; Akbulut, U.; Toppare, L. Conducting polymer composites of polypyrrole and polyindene. Synth. Met., 1996, 82, 4146.

[26] Selampinar, F.; Akbulut, U.; Toppare, L. Conducting polymer composites of polypyrrole and polyimide. Synth. Met., 1997, 84, 185-186.

[27] Yang, J.; Yang, Y.; Hou, J.; Zhang, X.; Xu, M. Polypyrrolepolypropylene composite films: preparation and properties. Polymer, 1996, 37, 793-798.

[28] Ouyang, M.; Chan, C.M. Conductive polymer composites prepared by polypyrrole-coated poly(vinyl chloride) powder: relationship between conductivity and surface morphology. Polymer, 1998, 39, 1857-1862.

[29] Aguilar-Hernandez, J.; Potje-Kamloth, K. Evaluation of the electrical conductivity of polypyrrole polymer composites. J. Phys. D Appl. Phys., 2001, 34, 1700-1711.

[30] Omastova, M.; Pavlinec, J.; Pionteck, J.; Simon, F.; Kosina, S. Chemical preparation and characterization of conductive poly(methyl methacrylate)/polypyrrole composites. Polymer, 1998, 39, 6559-6566.

[31] Boiteux, G.; Ho-Hoang, A.; Fache, F.; Glowaski, I.; Ulanski, J. Conductive composites based on PVDF- $\mathrm{C}_{2} \mathrm{~F}_{3} \mathrm{H}$ and subtituted or pure poly(pyrroles). Synth. Met., 1995, 69, 487-489.

[32] Mansouri, J.; Burford, R.P. Characterization of PVDF-PPy composite membranes. Polymer, 1997, 38, 6055-6069.

[33] Ryu, J.; Park, J.; Kim, B.; Park, J.O. Design and fabrication of a largely deformable sensorized polymer actuator. Biosens. Bioelectron., 2005, 21, 822-826.

[34] Misoska, V.; Price, W.E.; Ralph, S.F.; Wallace, G.G.; Ogata, N. Synthesis, characterisation and ion transport studies on polypyrrole/deoxyribonucleic acid conducting polymer membranes. Synth. Met., 2001, 123, 279-286.

[35] Yang, C.; Chen, J.; Swiegers, G.F.; Too, C.O.; Wallace, G.G. Hydrogen generation using PPy-FMS modified PVDF membrane and other substrates. Synth. Met., 2005, 154, 69-72.

[36] Gosh, M.; Barman, A.; Meikap, A.K.; De, S.K.; Chatterjee, S. Hopping transport in $\mathrm{HCl}$ doped conducting polyaniline. Phys. Lett. A, 1999, 260, 138-148.

[37] Nalwa, H.S. Evaluation of electrical conduction in iodine-doped polypyrrole. J. Mater. Sci., 1992, 27, 210-214.

[38] Coronado, E.; Gimenez-Saiz, C. A new BEDT-TTF salt and polypyrrole films containing the chiral polyoxometalate $\left[\mathrm{H}_{4} \mathrm{Co}_{2} \mathrm{Mo}_{10} \mathrm{O}_{38}\right]^{6-}$. Synth. Met., 2005, 154, 241-244.

[39] Mott, N.F.; Davis, E.A. Electronic Processes in Non-crystalline Materials. Oxford university Press, London, 1971.

[40] Bredas, J.L.; Yakushi, K.; Scott, J.C; Street, G.B. Polarons and bipolarons in polypyrrole: Evolution of the band structure and optical spectrum upon doping. Phys. Rev. B, 1984, 30, 1023-1025.

Received: October 30, 2008

Revised: November 10, 2008

Accepted: November 15, 2008

(C) Taunk et al.; Licensee Bentham Open.

This is an open access article licensed under the terms of the Creative Commons Attribution Non-Commercial License (http://creativecommons.org/licenses/by-nc/3.0/) which permits unrestricted, non-commercial use, distribution and reproduction in any medium, provided the work is properly cited. 